\title{
Recombinant Bispecific Single-Chain Antibody rM28
}

National Cancer Institute

\section{Source}

National Cancer Institute. Recombinant Bispecific Single-Chain Antibody rM28. NCI

Thesaurus. Code C60882.

A recombinant, bispecific, single-chain antibody directed against both the T-cell surfaceassociated costimulatory molecule CD28 and a melanoma-associated proteoglycan (MAPG) with potential antitumor activity. By targ eting both CD28 and MAPG, recombinant bispecific sing le-chain antibody rM28 enhances cytotoxic T-cell recognition of melanoma cells, which may result in immune effector cell-mediated tumor cell death and a decrease in distant metastases. This agent appears to have a long serum half-life secondary to the formation of dimers. When activated, CD28 facilitates interactions between T-cells and other immune effector cells resulting in cytotoxic T-lymphocyte responses; MAPG is a surface antigen expressed on the majority of melanomas, including primary cutaneous, ocular, and metastatic melanomas. 